\title{
A case of celiac disease mimicking amyotrophic lateral sclerosis
}

\author{
Martin R Turner, Gurjit Chohan, Gerardine Quaghebeur, Richard CD Greenhall, Marios Hadjivassiliou \\ and Kevin Talbot ${ }^{\star}$
}

\section{SUMMARY}

Background A 44-year-old male presented to a general neurology clinic with a 6-month history of progressive right-sided spastic hemiparesis without sensory symptoms or signs. The thigh muscle in the affected leg showed signs of wasting. The patient had a remote family history of celiac disease.

Investigations Neurological examination, neurophysiological studies, brain MRI scan, routine blood tests, duodenal biopsy, cerebrospinal fluid analysis including polymerase chain reaction test for JC virus DNA, serological testing for HIV and for the presence of serum antibodies to endomysium, gliadin and tissue transglutaminase.

Diagnosis Celiac disease with neurological involvement, mimicking amyotrophic lateral sclerosis.

Management Strict gluten-free diet.

KEYWORDS amyotrophic lateral sclerosis, antigliadin antibodies, celiac disease, hemiparesis, MRI

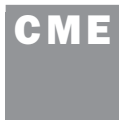

Vanderbilt Continuing Medical Education online This article offers the opportunity to earn one Category 1 credit toward the AMA Physician's Recognition Award.

\section{THE CASE}

A 44-year-old male was referred for a specialist neurological opinion with a 6-month history of progressive right leg weakness, and wasting and intermittent painful spasms of his right quadriceps. In the preceding month the patient had also noticed progressive weakness of his right arm and difficulty when writing. He had no sensory symptoms. The patient's only past medical history of note was migraine with aura. His family history revealed that a maternal aunt had celiac disease, a sister had Crohn's disease, and his maternal grandmother had multiple sclerosis. The referring neurologist had considered a provisional diagnosis of amyotrophic lateral sclerosis (ALS), but had not started the patient on riluzole.

Examination revealed right-sided spastic hemiparesis with a pyramidal pattern of leg weakness (Medical Research Council Grade 4-/5 in hip flexion, $4+/ 5$ in hip extension, $4 / 5$ in knee flexion and $4-/ 5$ in ankle dorsiflexion) associated with mild wasting of the right quadriceps. The patient had generalized bilateral hyperreflexia, sustained right ankle clonus and a right extensor plantar response. Results of cranial nerve, cerebellar and sensory examinations were normal.

$\mathrm{T}_{2}$ and fluid-attenuated inversion recovery brain MRI sequences revealed a region of hyperintensity along the course of the left corticospinal tract, arising from the subcortical white matter of the precentral gyrus and following the posterior limb of the internal capsule into the brainstem (Figure 1A). Gadolinium-enhanced MRI did not reveal any contrast enhancement. Repeat neuroimaging 2 months later revealed more-extensive changes in the same pattern, with additional involvement of the opposite (right) subcortical region of the precentral gyrus (Figure 1B).

Electromyography (EMG) of the masseter, biceps, first dorsal interosseous extensor digitorum 


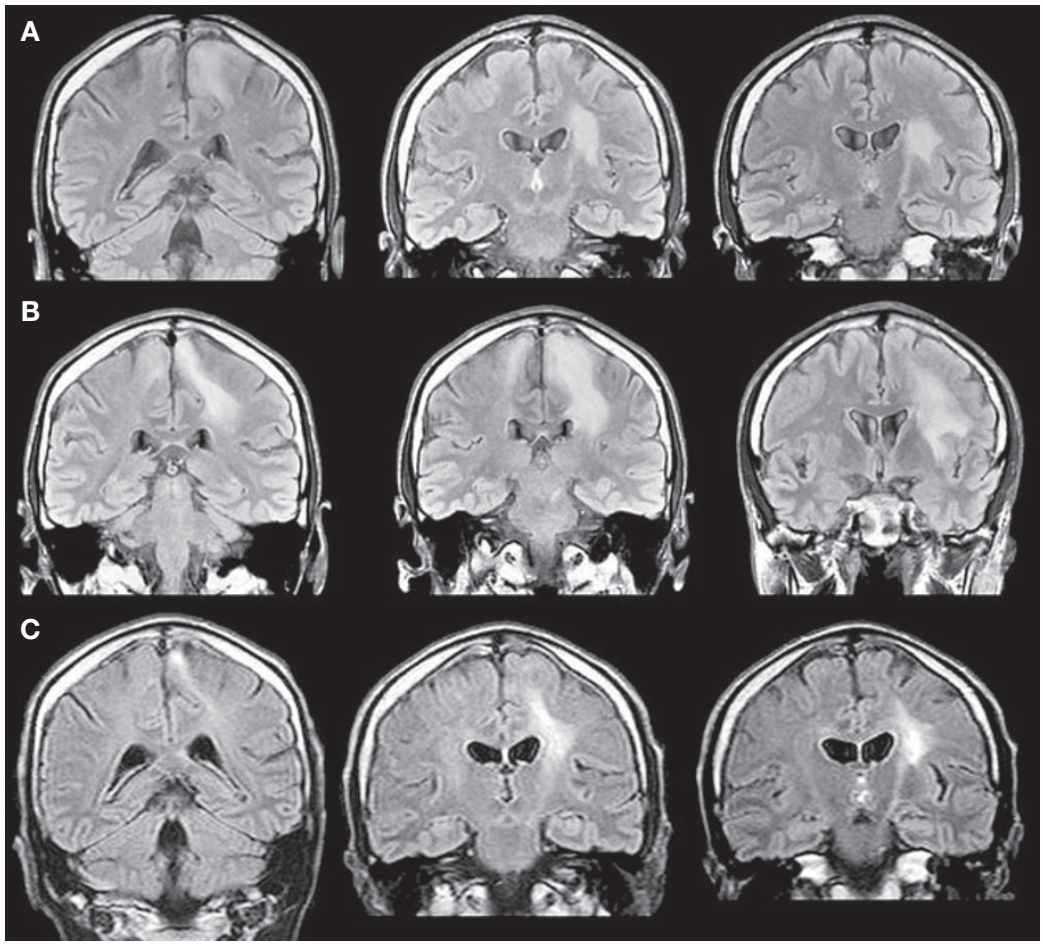

Figure 1 Coronal fluid-attenuated inversion recovery MRI of the patient's brain demonstrating regions of hyperintensity at initial presentation and 2 months later, with partial resolution following 9 months on a gluten-free diet. (A) Initially the hyperintensity is confined to the subcortical white matter of the left motor region, descending via the internal capsule into the brainstem. (B) Two months later there is more-extensive hyperintensity visible in the left hemisphere, and limited involvement of the right motor cortex. (C) After 9 months on a gluten-free diet, the hyperintensity of the left corticospinal tract is more confined, and the right motor cortical changes have resolved. Neuroradiological improvement was accompanied by substantial clinical improvement of the patient's right-sided hemiparesis.

communis and the vastus medialis muscles demonstrated widespread fasciculations, reduced recruitment of motor units, and frequent complex polyphasic waveforms. Fibrillation potentials were recorded in the right vastus lateralis. Results of nerve conduction studies were normal. Cerebrospinal fluid analysis revealed that all constituents were normal and a polymerase chain reaction test for JC virus was negative.

Routine blood tests revealed a mild microcytic anemia (hemoglobin $129 \mathrm{~g} / \mathrm{l}$, normal range 120$140 \mathrm{~g} / \mathrm{l}$; mean corpuscular volume $78 \mathrm{fl}$, normal range $80-98 \mathrm{fl}$ ) with low levels of serum iron $(3.5 \mu \mathrm{mol} / \mathrm{l}[20 \mathrm{~g} / \mathrm{dl}]$, normal range $45-300 \mu \mathrm{mol} / \mathrm{l}$ [251-1,676 g/dl]), serum ferritin (17 pmol/l; normal range $45-675 \mathrm{pmol} / \mathrm{l}$ ) and serum folate $(8.6 \mathrm{nmol} / \mathrm{l}$, normal range $7-40 \mathrm{nmol} / \mathrm{l})$. Results of a subsequent antiendomysial antibody test were positive (IgA level $5.88 \mathrm{~g} / \mathrm{l}$ ). Duodenal biopsy analysis demonstrated villous atrophy, crypt hyperplasia and increased intraepithelial lymphocytes (Marsh 3A), consistent with gluten-sensitive enteropathy (celiac disease). Tests for HIV type 1 and 2 antibodies were negative.

The patient was started on a gluten-free diet approximately 7 months after the onset of his initial neurological symptoms. No drugs, including riluzole or other agents with neuroprotective potential, were given. At a follow-up examination 9 months after initiation of treatment, the patient's right arm function, assessed by the neurologist who performed the initial examination, had returned to normal. Improvement in the patient's right leg function was more limited, wasting was still present and there was some residual spasticity. He was now able to walk unaided, however, and his handwriting and ability to fasten buttons had returned to normal. Repeat MRI demonstrated partial resolution of the corticospinal tract changes (Figure 1C). EMG examination was not repeated after treatment. The patient's antigliadin antibody response fell from pretreatment levels of IgA $34 \mathrm{U} / \mathrm{ml}$ and $\operatorname{IgG} 44 \mathrm{U} / \mathrm{ml}$ (normal $<15 \mathrm{U} / \mathrm{ml}$ for both) to $\operatorname{IgA} 18 \mathrm{U} / \mathrm{ml}$ and no detectable $\operatorname{IgG}$ antibody. The patient was actively followed up for 2.5 years after his initial presentation, with no evidence of neurological relapse.

\section{DISCUSSION OF DIAGNOSIS}

In this Case Study, the patient's initial presentation consisted of a progressive motor syndrome in the absence of sensory signs, with clinical evidence of upper and lower motor neuron degeneration, electromyographic evidence of widespread acute denervation, and hyperintensity in the corticospinal tracts revealed by MRI. In view of these findings, while acknowledging the unusual hemiparetic presentation and strikingly territorial nature of the white matter changes seen on MRI, a diagnosis of amyotrophic lateral sclerosis (ALS) was initially considered by the referring neurologist. The apparent presentation of a rare ALS variant in association with celiac disease-a condition with various neurological manifestations - made it entirely appropriate, however, to review the initial diagnosis and institute treatment for celiac disease. Ultimately, improvement in the patient's symptoms following treatment for celiac disease rendered the diagnosis of ALS untenable.

Screening for celiac disease was prompted by the discovery of microcytic anemia with low serum iron and folate levels. Antiendomysial antibodies-a highly sensitive and specific marker for gluten-sensitive enteropathy-were 
detected. The confirmatory duodenal biopsy analysis demonstrated the triad of villous atrophy, crypt hyperplasia and increase in the number of intraepithelial lymphocytes that characterizes celiac disease. IgG and IgA antigliadin antibodies and anti-tissue transglutaminase antibodies were also detected - these have greater sensitivity for extraintestinal manifestations of celiac disease than do antiendomysial antibodies. ${ }^{1}$

Celiac disease is an immune-mediated systemic disease. For every patient presenting with the classic symptoms of gastrointestinal involvement and malabsorption, there are an estimated eight patients with 'silent' disease or with extraintestinal manifestations. Celiac disease is prevalent in 1 in 100 individuals in most European countries and in the US. ${ }^{2}$ The pathological trigger is gluten, a protein commonly found in rye, barley and wheat. Celiac disease has a strong hereditary component: its prevalence in first-degree relatives ranges from $10-20 \%{ }^{3}$ The human leukocyte antigen DQ2 is present in $90 \%$ of cases.

The observation that celiac disease is associated with a broad range of neurological symptoms was first made over 40 years ago. ${ }^{4}$ The most common of these neurological complications are ataxia and neuropathy, ${ }^{5}$ including pure motor variants, which have been reported to have EMG changes consistent with motor neuron disease. ${ }^{6}$ There was no evidence of ataxia or peripheral neuropathy, however, in the present case. Celiac disease can also be associated with seizures, dementia and myopathy. White matter changes associated with gluten sensitivity have been variously described as a form of multifocal leukoencephalopathy or as a more patchy process associated with prominent headache. ${ }^{7}$ To our knowledge, a syndrome of progressive hemiparesis with such striking changes to the corticospinal tract MRI as were seen in the present patient has not previously been reported in association with celiac disease.

The mechanism of neuronal damage in celiacassociated neurological disease is still unclear. One hypothesis is that perivascular inflammation might lead to the breakdown of the blood-brain barrier, allowing an influx into the brain of antibodies that cross-react with neural tissue. The arterial wall contains high levels of transglutaminase, and IgA deposits against transglutaminase 2 within the arterial wall of vessels from the cerebellum have previously been detected in a case of gluten ataxia. Anti-transglutaminase-2 IgA deposits in the gut have also been reported and they are considered to be the earliest markers of gluten sensitivity. ${ }^{8}$

\section{DIFFERENTIAL DIAGNOSIS}

This patient presented with symptoms consistent with ALS, although there were atypical features that suggested that other diagnoses needed to be excluded. ALS is a neurodegenerative condition of motor neurons with a consistent worldwide incidence of approximately 1-2 cases per 100,000 individuals per year, and a 3:2 male predominance. The majority (90-95\%) of cases are apparently sporadic, and less than $2 \%$ of the total number of cases are associated with an underlying mutation of the superoxide dismutase 1 (SOD1) gene. The etiology of ALS is unknown, although there are multiple molecular hypotheses to explain the specificity of motor neuron degeneration. ${ }^{9}$

Despite anecdotal reports of exceptional cases, ALS is a condition with relentless progression, and riluzole, the single disease-modifying drug licensed for the condition, provides only an approximately $10 \%$ improvement in mean survival in a trial setting. Although around 5\% of all patients with motor neuron disease, particularly but not exclusively those with primary lateral sclerosis, survive well into a second decade from symptom onset, periods of arrested disease progression or obvious improvement of symptoms are not observed during long-term follow-up in specialist clinics. ${ }^{10}$

As there is currently no cure for ALS and the mean survival time from symptom onset is 34 years, it is important to consider all potentially treatable diseases that might produce a similar syndrome. Clinicians should recognize, however, that in practice such 'mimics', although understandably appealing to anxious patients and their carers, are extremely rare.

The role of neuroimaging in the investigation of suspected ALS is most frequently to exclude cervical spine pathology, which can present clinically with upper and lower motor neuron signs in the limbs. In this case MRI conclusively excluded compressive pathology. Cerebral MRI can also be useful in patients with bulbar dysfunction to exclude an alternative cause such as stroke, demyelination or a brain stem tumor. Although hyperintensity of the corticospinal tracts on MRI-particularly with fluidattenuated inversion recovery sequences-is a well established phenomenon in ALS cases, ${ }^{11}$ this sign has low diagnostic sensitivity and specificity. The hyperintensity seen in the present case, which was particularly extensive in the region of the precentral gyrus, showed an unusual degree of confluence. 
Competing interests

The authors declared no competing interests.
The lesions in the present case were revealed by MRI to have some features in common with those seen in progressive multifocal leukoencephalopathy. The effects of progressive multifocal leukoencephalopathy are usually widespread, but a case has been described in which the lesions were confined to the pyramidal tract. ${ }^{12}$ For this reason, the present patient was tested for HIV and JC virus, with both tests being negative.

EMG can provide supportive evidence in the investigation of a case of suspected ALS, but it is only diagnostic in the context of a compatible clinical history and examination. The value of EMG in the investigation of ALS lies in the detection of subclinical lower motor neuron involvement in seemingly asymptomatic muscles, which could suggest a widespread degenerative process rather than focal denervation. Although changes suggestive of widespread denervation were observed in this patient, they were not of sufficient magnitude to enable a diagnosis of ALS to be made on purely electrophysiological grounds.

\section{TREATMENT AND MANAGEMENT}

In published reports of neurological complications of celiac disease, improvement with dietary modification has not been consistently reported, and the association of these complications with celiac disease consequently attracts controversy. Lack of improvement might be the result of unintentional poor dietary compliance, caused by variable food labeling practices and the fact that even minute traces of gluten, enough to perpetuate the immune response, can persist on cooking implements. The benefits of a gluten-free diet for patients with ataxia and neuropathy caused by gluten sensitivity have been demonstrated in systematic studies that closely monitored dietary adherence via regular assessment of antigliadin antibodies. ${ }^{13}$

Levels of antiendomysial, anti-tissue transglutaminase and antigliadin antibodies should diminish following avoidance of gluten, so incomplete recovery in the presence of persistently positive tests for these antibodies should prompt a thorough review of the patient's diet. Gluten-free products contain wheat that has been processed to remove most, but not all, of the gluten. For most patients, such small amounts of gluten are innocuous. For some very sensitive patients, however, even traces of gluten can be enough to perpetuate an immunological response, as indicated by the persistence of symptoms and positive tests for the relevant serological markers. In such cases a 'wheat-free' diet — a diet that avoids wheat completely and uses alternative sources of protein such as rice or corn flour-can be beneficial. As a result of the detection of residual antigliadin IgA levels, the patient discussed here is currently following a wheat-free diet.

\section{CONCLUSIONS}

The patient discussed in this Case Study presented with a syndrome of progressive hemiparesis associated with gluten-sensitive enteropathy. The clear clinical and neuroradiological improvements that followed treatment with a gluten-free diet are evidence that the syndrome of progressive hemiparesis represents another addition to the growing list of neurological manifestations of celiac disease.

The symptoms displayed by the patient in this case were initially suspected to result from ALS. This condition remains predominantly a clinical diagnosis, and the aim of diagnostic investigations is the exclusion of alternative 'mimic' syndromes that might be responsive to treatment. ALS is a condition with relentless progression; for this reason, the simple observation of an improvement in symptoms is most pertinent in rendering the diagnosis of ALS untenable.

\section{References}

1 Hadjivassiliou Met al. (2004) The immunology of gluten sensitivity: beyond the gut. Trends Immunol 25: 278-282

2 Sanders DS et al. (2003) A primary care cross-sectional study of undiagnosed adult celiac disease. Eur J Gastroenterol Hepatol 15: 407-413

3 Esteve M et al. (2006) Spectrum of gluten-sensitive enteropathy in first-degree relatives of patients with coeliac disease: clinical relevance of lymphocytic enteritis. Gut 55: 1739-1745.

4 Cooke WT and Smith WT (1966) Neurological disorders associated with adult celiac disease. Brain 89: 683-722.

5 Hadjivassiliou M et al. (1998) Clinical, radiological, neurophysiological, and neuropathological characteristics of gluten ataxia. Lancet 352: 1582-1585

6 Hadjivassiliou M et al. (2006) Neuropathy associated with gluten sensitivity. J Neurol Neurosurg Psychiatry 77: 1262-1266

7 Hadjivassiliou M et al. (2001) Headache and CNS white matter abnormalities associated with gluten sensitivity. Neurology 56: 385-388

8 Hadjivassiliou M et al. (2006) Autoantibody targeting of brain and intestinal transglutaminase in gluten ataxia. Neurology 66: 373-377

9 Boillee S et al. (2006) ALS: a disease of motor neurons and their non-neuronal neighbors. Neuron 52: 39-59

10 Turner MR et al. (2003) Prolonged survival in motor neuron disease: a descriptive study of the King's database 1990-2002. J Neurol Neurosurg Psychiatry 74: 995-997

11 Hecht MJ et al. (2001) MRI-FLAIR images of the head show corticospinal tract alterations in ALS patients more frequently than T2-, T1- and proton-densityweighted images. J Neurol Sci 186: 37-44

12 Sobha N et al. (2005) Progressive multifocal leucoencephalopathy with discrete involvement of pyramidal tract. J Neurol Neurosurg Psychiatry 76: 24

13 Hadjivassiliou M et al. (2006) Dietary treatment of gluten neuropathy. Muscle Nerve 34: 762-766 